\title{
An Information model of Interagency Communication Based on Distributed Data Storage
}

\author{
Yuri P. Lipuntsov \\ Associate professor, Department of Economics, Lomonosov Moscow State University, \\ Leninskie Gory 1/46, Moscow, 119992, Russia \\ tel: +007(495)9395708 \\ lipuntsov@econ.msu.ru
}

\begin{abstract}
The organization of interagency communication is largely determined by accepted practice from the information model, by the principles which are used for the development of standards for information exchange and by the methods used to bring government reports into conformance with those standards. In world practice various models are applied. This article presents a comparative analysis of these models and proposes a variation of the information model that is based on distributed data storage. The proposed model answers the needs for flexibility and expandability, and also offers an opportunity to distribute responsibility and ensure a sufficient level of security of government data. The basis for interagency communication in the proposed model is a three-layer data hub, including a general hub, an object field hub, and an object-oriented portion of the hub. Horizontal and vertical connection among the separate parts of the hub is accomplished by means of a model of intermodel connections.
\end{abstract}

\section{CCS Concepts}

- Information systems Database design and models. - Information systems Data exchange. Information systems $\sim$ Data warehouses.

\section{Keywords}

"Information modeling"; "Data exchange"; "Shared environment”; "Data warehouses”; "Domain data model”

\section{INTRODUCTION}

The success of ICT projects in the government sector of data processing is largely determined by the level of data processing. A feature of data processing in the government sector is the shared use of the data that are collected in agency systems but are used in the performance of services of various sorts and conducting government functions. In many texts, including government documents, the theme of shared used of data and a more active use of the information assets that have been gathered is often stressed. In this connection the integration technologies remain behind the scenes and are taken to be a previously solved problem [1]. This could turn out to be a simple task, but most agency

Permission to make digital or hard copies of all or part of this work for personal or classroom use is granted without fee provided that copies are not made or distributed for profit or commercial advantage and that copies bear this notice and the full citation on the first page. Copyrights for components of this work owned by others than ACM must be honored. Abstracting with credit is permitted. To copy otherwise, or republish, to post on servers or to redistribute to lists, requires prior specific permission and/or a fee. Request permissions from Permissions@acm.org.

EGOSE '16, November 22-23, 2016, St.Petersburg, Russian Federation

(C) 2016 ACM. ISBN 978-1-4503-4859-1/16/11 ...\$15.00

DOI: http://dx.doi.org/10.1145/3014087.3014097 systems, in which huge amounts of data have been collected, are focused on solving local problems and are not amenable to integration with other systems. The problem of shared use of data becomes more and more complex with the extensive use of mobile devices, new types of data, and the constant collection and updating of data.

The approach described herein is characteristic for the adopted variant of a System design of electronic government in the Russian Federation, reflecting the general principles of architecture development, one of the layers of which is the defined layer "Information and Data" [2]. Other documents [3] governing the creation of informational communication models, also do not contain a definitive description of principles but are based on the development of existing systems of interagency communication, not presupposing the creation of a single system of standardization for data exchange. Creation of an information model for information exchange within an electronic government is a current task for Russia. Development of a strategy, methodology and tools are necessary for the effective use of government data. The active use of collected information assets, allowing access to data, will open new possibilities for innovation, involving active citizens in government management, and increasing its success rate.

\section{THE INFORMATION MODEL}

The foundation for data processing is the information model, which determines the basic principles for organizing the exchange of data among systems. The information model that is used in government activity should provide delivery of current data about various objects found in the field of government management with necessary attributes, the content of which is determined by the context of the process.

It is expedient to organize data processing within an electronic government on industrial principles. The industrialization of data processing presupposes such methods as standardization of principles for creating data models, assignment of responsibility, and increasing skills in the field of data processing.

The task of creating an information model for interagency communication can be defined as the creation of an infrastructure model for using single elements of data independent of the number of sources of the required information in rendering services to citizens, business, and administration.

The government sector requires the development of an information model that would provide proper data processing at least in the mid-term. The information model should provide access to government data when that is necessary and with sufficient protection of private life and security of valuable government data. 
Some part of the data can be presented in the "open data" format and other data processing requirements place a whole number of requirements in order to solve a set of data processing problems as a single approach. A strategy in the field of data within an electronic government on the one hand entails the use of digitized data and on the other hand the use of a policy of data openness on the part of departments, in order to make the data more accessible for the public and to attract citizens to examine the data and analyze them, including in the context of one's family income, personal hobbies, consumer particulars, etc.

One of the important aspects when creating an information model is to determine the boundaries of the system, in particular, what portion of the data will be included in the system's realm of control, that is, will be transformed and modified for populating the system with information. When creating integration systems, the desire often arises not to transform the source data that exists within the systems in operation.

The informational data exchange environment presupposes acceptance of certain standards, including in the field of information and data. That means that data from local systems must be transformed and brought into conformity with those standards. Various transformation technologies can be used for this purpose.

\section{GENERAL REPRESENTATION OF INFORMATION COMMUNICATION}

The activity model that reflects the participants in a process and their actions is a basis for the development of an information model. Such elements as the processes of the activity and data exchange sessions in implementation of that process are described in the framework of the model. In the most basic version the activity model can be viewed as a compilation of three elements:

- Agents of the communication

- Communication among the agents

- Information circulating during the communication

The agents of the information communication may act in the capacity of data providers or consumers.

The primary consumers of data are citizens, representatives of business, representatives of social organizations, government and local government authorities, and other state organizations with government management authority. The data providers are often organized participants: government agencies or business. The concept of electronic government includes the activity of all branches of government, including not only the executive, but also the legislative and judicial branches. The classifiers of the functions and authorities of the government bodies are an important part of the activity model. The functions and authorities of the government bodies are difficult to describe in the form of a flat structure; therefore a methodology is formulated for systematizing the functions and authorities. This methodology assumes the construction of a multidimensional model. Government lists and registers are considered as the basic information of such a model and its implementation in the form of classifiers [4].

With increased informational maturity, opportunities for offering services grow: from informational and communications interactions one can shift to transactional and interactive activities [5], which assume multiple interactions in the framework of a single service, as well as being oriented to obtaining information from users, including in the form of feed back. A client-oriented approach assumes the use of the tools of proactive services furnished for situations in life and the segmentation of users in order to deliver an individual set of services. The government creates an information environment that expands citizens' opportunities and allows them to take an active part in the processes of government management, to have a vote in the decision-making process.

Creation of an information model presupposes regulation by several categories of information resources, including providing information turnover in the framework of the regulated processes, standardization for offering services on websites, and portals of agencies and other participants in the information exchange. Besides, another important aspect is the direction of applying a single methodology for establishing data models in the systems to be developed, and adopting templates for specific object fields, which could be an initial version for standardizing data for local systems.

The information model should presuppose the development of integrational mechanisms for the information systems of all branches of government with infrastructure elements of electronic government, including a system for exchanging reports, a system of identification and authentication, a portal for government services, and a single personal office.

\section{THE PRINCIPLES FOR BUILDING AN INFORMATION MODEL AT THE NATIONAL LEVEL}

A significant portion of the processes of government management bears an interagency character, and implementing them in electronic form presupposes an active information exchange. The information model is a basis for implementing information exchange. An information model can be constructed on various principles, which predetermine their basic characteristics.

The first important question that arises when constructing an information model of communication is the character of the exchange: the exchange can be accomplished either through a central hub or on a point-to-point principle.

If a model through a central hub is chosen, the number of junctions is reduced to $\mathrm{N}$, while in the point-to-point version the number of junctions is far greater. These two versions differ in the possibilities for managing processes and implementing new models, as well as such characteristics as flexibility when expanding by function and scale. When using a model with a central hub it is easier to exert control over the communication system. In the centralized structure it is simpler to implement new models and expand existing ones, but in doing so system flexibility is reduced from the point of view of the possibility of implementing an initiative from below.

A second basic question is the format for storing the transferred information. In this case the term "information" is understood to include information about basic informational objects, both general for all object fields (natural persons, legal persons, an address system, and others) as well as objects of the subject field (medicine, education, etc.).

Let us examine the procedure for forming an exchange packet as a third supplemental characteristic of systems. As variations for comparison of various governmental information exchange models, we will consider models implemented in the European Union, America, and Japan, will compare those with the existing model and a model proposed by recent state documents. 
In the European Union, for providing pan-European electronic services, the ADMS [6] family of models is being developed; this group presupposes the creation of a network of repositories. The information assets meeting the standards are loaded into such repositories. The standards are placing requirements for data formats and metadata describing the data. Semantic web technologies assuming adoption of unique identifiers for specific information assets are used for organizing communication among the various sets of data.

The United States uses the NIEM [7] model, which consists of data formats for specific object fields. The IEPD exchange package is used for data exchange. The data for preparing a package is taken from local systems. Data for exchange are prepared by the data supplier. Currently, a group working to implement the NIEM ideology is considering a variant of creating a system for information exchange; that is, prospectively data exchange through a central hub is being proposed.

An intermediate version for exchanging government data is being developed in Japan [8]. This version assumes the creation of a

Table 1 National scale infor facility for storing current data as related to a universal data hub and object field hub on the one hand while obtaining data from local systems as related to an object-oriented fiend on the other hand.

In the Russian Federation, in the infrastructure of the electronic government [9], including the Interagency Electronic Communication System (IECS), the Unified System of Identification and Authentication (USIA), and the Unified Portal of State and Municipal Services (UPSMS), the exchange of data occurs through a central hub. Data are stored in local systems and are presented for exchange with the use of web service technologies; data are described by means of process charts. Recent documents in the field of information circulation regulation assume the creation of some sort of system of standardization of information circulation. The system herein proposed focuses on creation of a Unified Data Model.

The table below presents a comparison of the information models used in electronic government (EG).

\begin{tabular}{|c|c|c|c|c|}
\hline & NIEM & ADMS & IMI & EG Infrastructure \\
\hline $\begin{array}{l}\text { Presence of central hub (Hub, no } \\
\text { hub) }\end{array}$ & No hub & Hub & Hub & Hub \\
\hline Preparation of data for exchange & $\begin{array}{l}\text { Adaptation to } \\
\text { standard on request }\end{array}$ & $\begin{array}{l}\text { Adaptation to standard } \\
\text { during loading }\end{array}$ & $\begin{array}{l}\text { Loaded data are } \\
\text { standard-ized }\end{array}$ & $\begin{array}{l}\text { Description of data } \\
\text { available in local } \\
\text { systems }\end{array}$ \\
\hline Area of standardization & $\begin{array}{l}\text { Format of data for } \\
\text { exchange }\end{array}$ & Format of data storage & $\begin{array}{l}\text { Data format/ } \\
\text { semantic model }\end{array}$ & \\
\hline Location of storage of source data & Local IS & Repository & $\begin{array}{ll}\text { Local } & \text { IS/ } \\
\text { Repository } & \end{array}$ & Local IS \\
\hline $\begin{array}{l}\text { Technologies of } \\
\text { documents }\end{array}$ & IEPD & RADion & IEP & Process charts \\
\hline
\end{tabular}

NIEM — National Information Exchange Model (USA)

IEPD — Information Exchange Package Documentation (USA)

ADMS - Asset Description Metadata Scheme (EU)

RADion - Repository Asset Description (EU)

IMI — Infrastructure for Multilayer Interoperability (Japan)

IEP — Information Exchange Package (Japan)

EG Infrastructure — system of interagency electronic communication (Russia)

From the presented review of communications variations in the European, American, and Japanese experience, the boundaries of the system in which standardized data fall have been defined. In the case of the European Union and Japan they adapted to a standard when loading into the system, while in the American version they are transformed into XML, meeting the requirements of a standard when a user makes a query. In the version laid out in Russian government documents, a version is presented that assumes the creation of a Unified Data Model, describing the data to be stored in local systems but does not assume their transformation for use in the framework of an information exchange. Such a scheme turns out to be difficult to implement, since on the one hand a unified model must be developed, which unites all interrelated systems participating in supplying data, and on the other hand it provides a flexible mechanism for modernizing the unified model when migrating a local system data model.

\section{VARIATIONS ON TRANSFORMING LOCAL SYSTEM DATA TO AN EXCHANGE FORMAT STANDARD}

Data must be perceived uniformly by all participants of information exchange in order to ensure proper information exchange. Two variations are possible here: In the first variation, data from local systems are transformed into the data set conforming to the standard when there is a query from the data consumer.

In the second variation, all data from local systems that might be demanded in the framework of specific processes are transformed and loaded into some sort of storage facility, which might have a distributed structure.

The first variation for transformation can be implemented using the CAM 3.0 tool, which is the result of the Verify XML 
initiative. This initiative offers a set of tools with open source code for creating a component for exchanging data with the use of framework standards of data formats. One of the scenarios for using CAM is support of the NIEM, used for data exchange in government management in the United States. The operation of the aggregate of components presupposes delivery of data from legacy systems in the XML format. Data are transformed into the format of standardized data, in particular into the NIEM format for data exchange, and are also verified for compliance with the standards.

As an example of using this transformation and data verification tool, a prototype application was shown that enables the users to find a needed medication in pharmacies in Chicago. The application transforms the data from SQL into the corresponding NIEM XML standard, and those data are further transformed into card format. In the framework of the Government 2.0 seminar, the use of that tool for delivering data about university pedagogical activity in the format of a conventional standard for the subject field "Higher Education" [11] was demonstrated.

The second variation is creation of a data storage facility in which the necessary data are collected. The data are brought to accepted standards when the data are loaded, and besides are supplied with metadata for describing the data. The models of integration that use the storage facility as a data exchange node can be roughly divided into two classes: the hypercube, or the Data Vault model [12], on the basis of which lies a chart of the basic entities and the connections between them.

The hypercube consists of a multidimensional generalization of a two- or three-dimensional table for a multidimensional data set, with the assumption that data can have an arbitrary number of dimensions. For example, at the regional level, calculation of financial data is possible based on projects, on periods of time, on subjects, etc., with the purpose of comparing actual or budgeted expenditures. Each cell of the cube will have a number representing a certain value that reflects the facts of the activity, such as expenditures, budget, prognosis, etc.

In the case of a hypercube, data are usually stored in the form of a diagram - a star or snowflake in relational database management systems or in special-purpose database management systems. Values are stored in a central table of facts, and various measurements are described in the form of directories, specific ones of which may have a hierarchical structure. The hypercube model is used for organizing data storage in SDMX. An analogous model is taken as the basis for organizing data storage in ADMS.

In contrast to the hypercube, the Data Vault represents a method for modeling databases that is intended for storage of data coming from several transaction systems incl. tracking of all changes occurring in local systems. This is achieved by virtue of the fact that the data storage model has a two-level structure: On one level the basic components are presented with the connections between them, while on the second level the storage of data is organized. Such a storage model makes it possible not only to look through current and historical data but also to implement such data management characteristics as the robustness of the model to change and its expandability, as well as to conduct a data audit and check the speed of their loading and other factors related to database maintenance.
By using this technology it is possible to create database kernels as a set of data that provide informational interaction between agencies and systems. The kernel fulfills the function of collecting and comparing data about general basic information objects and basic information objects of subject fields coming from local sources.

\section{THE MODEL OF INTERAGENCY INTERACTION BASED ON DISTRIBUTED STORAGE}

The interagency interaction that arises when administrative processes are implemented is usually limited to an exchange of pieces of information reflecting the state of basic information objects. Storage of current data acts in that case as a block of data aggregating a set of pieces of information that are needed for exchange. The model of the kernel will allow one to compare information about analogous objects coming from various systems, and to track the dynamics of their state. The data model of the kernel may have a description of semantic connections between objects, which will allow to implement the semantics using the context. Based on the kernel model it is possible to organize the Benchmark Data module about basic objects and the Data Showcase module.

The model of information and data for arranging an information exchange in the framework of electronic government assumes the capture of a sufficiently broad spectrum of directions of government activity. Taking this into account, the model should contain several sections, which will be distinguished by the nature of the assignment to specific subject fields. A data kernel, as a basis for interagency interaction, consists of a multilayered structure. As foreign experience shows, the kernel can include two (US, EU) or three (Japan) layers. The general kernel contains a set of universal components that are used in all the subject fields. The universal kernel in the European Union includes four components: natural persons, legal persons, location, and a catalogue of services. The same list in Japan and the US is broader and includes other elements, such as goods, capacities, etc.

In this case we will examine the three-layered structure of a data kernel: the general kernel, the kernel of the subject field, and the subject-oriented portion of the kernel. Interaction among the layers will occur using the model of interlevel communications.

\subsection{The general kernel}

The general data kernel includes the universal portion of the data that are used in many subject fields are understood in a uniform manner by them. In the prevailing practice of managing information resources [3], the general kernel may include data on natural persons, legal persons, a description of location, real estate items, and possibly other objects.

The following figure presents a version for organizing storage of General kernel data, including four basic entities: natural persons, legal persons, location, and real estate items. The model allows to organize storage of data loaded from various systems and to receive a selection of data by various layers: citizenship, place of residence, registration, date of updating, etc. 


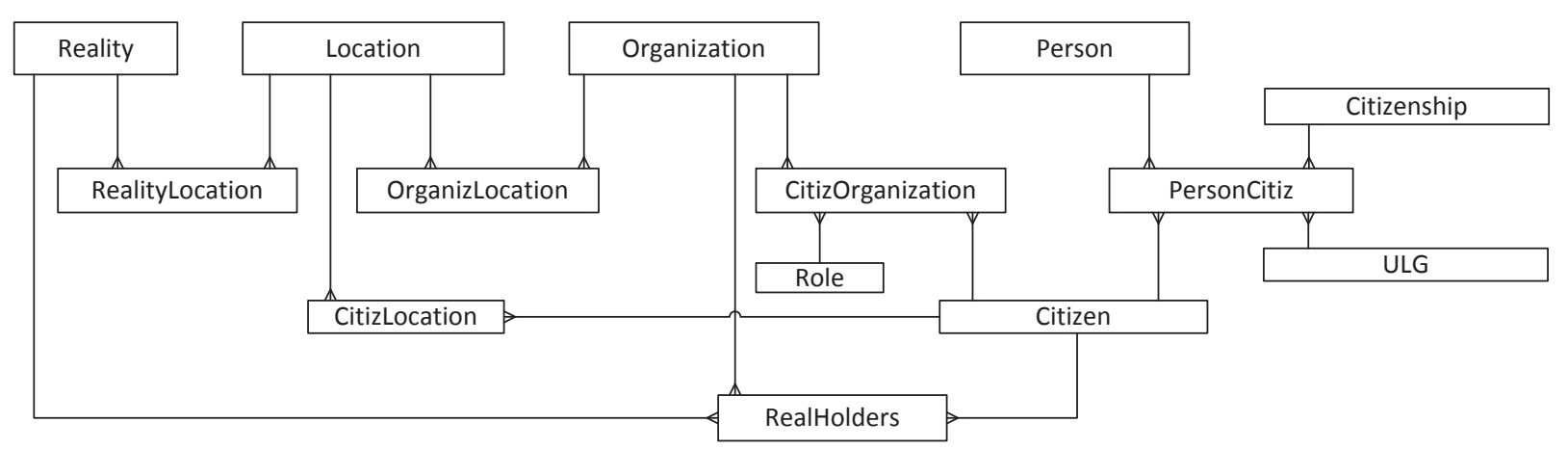

Figure 1. Version of codifier content of the data general kernel

The content of general kernel data is determined by the content of the tasks to be solved. In Japan, where tasks of government supply are solved by means of this kernel, the data content of the universal kernel includes goods, contracts, a chart of deliveries, etc.

The universal kernel is the basis for information exchange of the subject field. Data entering the data kernel pass through data processing, as a result of which the basic entities are encoded in accordance with the ontology of the subject field. Encoding is performed in such a way that all identical objects entering the system from various sources receive one code. Such a system of encoding makes it possible to examine the reflection of a particular object in various systems. Upon loading into the system, the entry shows the source of the data, the time of loading, and the time of expiry of data timeliness.

\subsection{Subject field kernel}

The subject field kernel is an expansion of the general kernel. The subject field as a field of government activity is divided into a subset of directions. The contents of the subject fields is predetermined by the level of informational maturity of the branch, as well as by other factors, such as the presence of a query from a client, the infrastructure, etc.

The subject field model includes

Basic entities - information objects of the subject field, and connections among them;

A graphic presentation of the subject field - a hierarchical presentation of objects of the subject field, which allows to construct a relationship of objects in the form of a real world model;

Supplemental information resources include Classifiers, Directories, and Intersubject code lists.

The model of the subject field describes the aggregate of the objects which are under control of a specific subject field and consists of analogous blocks: the basic components of the subject field as an aggregate of the entities necessary for a sufficient description of the subject field, the connection of the entities of the subject field.

Let us examine a conceptual description of a subject field in more detail using the example of the "Higher Education" sector (Fig. 2). Other examples could be: Oncology, Defective Products, Driver’s Licenses, etc.

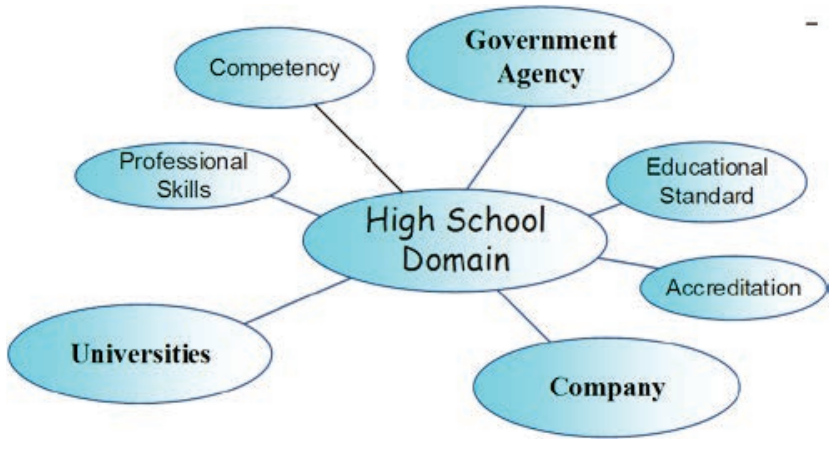

Figure 2. Basic entities for describing the subject field Higher Education

\subsection{Subject-oriented kernel}

Fulfilling functions presupposes a detailed description of the activity. Let us examine the subject-oriented kernel using an example of the Higher educational institution. An educational institution entails such concepts as the Training Program, the Teaching Plan of the programs, a Student Group, Discipline, Resources of specific disciplines, Skills, etc.

The ontology of the subject field is a basis for creating the data model of this type of storage. The methodology for compiling the ontology for data storage is described in the author's work "Principles of Creating an Ontology for Data Storage. The Example "Higher Education" [13]. The methodology includes dividing all entities into primary and derivative. Each of the entities has a content key; the keys of derivative entities are formed as composites of the keys of primary entities. This allows to create keys for transactions containing activity logic in terms of the primary entities.

In describing the subject field "Higher Educational Institutions", the following concepts fall into the category of concepts for formulating the primary entities, each defined independently: Enrolment, Program, Group, Persons, Subdivisions, Discipline, Period, Academic Year, Type of lessons, Classes (lasting 2 academic hours), Date, Lecture hall. The following set of concepts is defined using other concepts, therefore it will be used for constructing the derivative entities: Enrolment for a Program, Student Group, Student, Colleague, Course, Stream, Class. A graphic presentation is given in Fig. 3. 


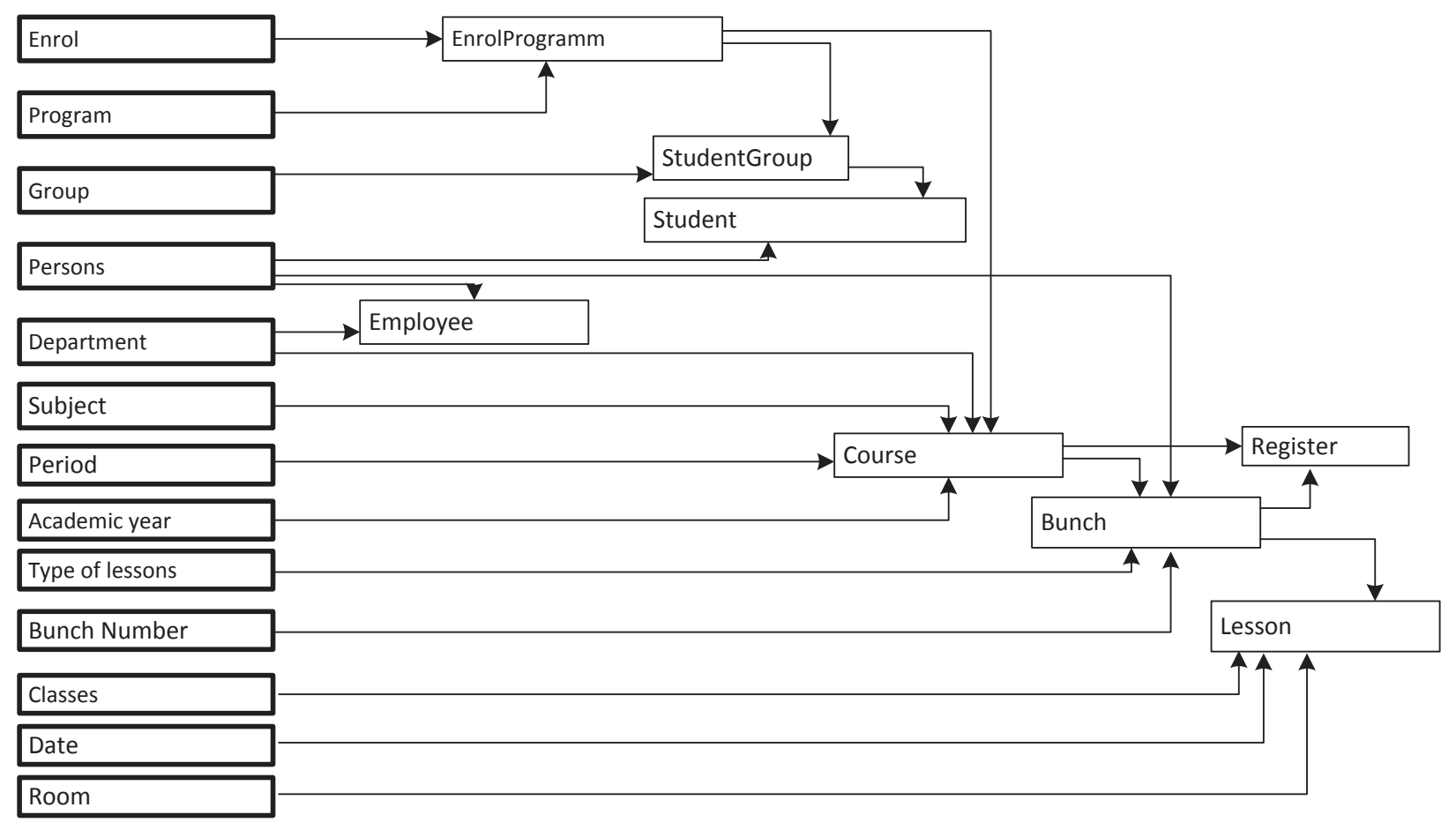

Figure 3. Graphic presentation of the subject field Pedagogical Activity of the educational institution of the Higher Educational Institution

\subsection{Model of interlevel interactions}

Agreement between separate models, both on the vertical and on the horizontal levels, is an important element in organizing interagency interaction. In order to organize the connections, a model of interlevel interactions is created, which provides agreement among the object presentations in the various models. Variations of agreement could include creation of a mechanism for using a single object codifier, or establishing coordination among the object presentations in the various models. Figure 4 shows a version of agreement among a universal kernel, a subject field kernel, and the subject-oriented portion of the description. The kernel data model is divided into two parts: frame and data. The frame consists of a set of entities containing identifiers of the basic objects and their connections. Data about objects and transactions with them are presented separately and can be implemented in the form of links to data sources; i.e., the kernel data have a distributed structure and can be found in the responsibility field of the local operator systems, from which data are delivered for interagency exchange.

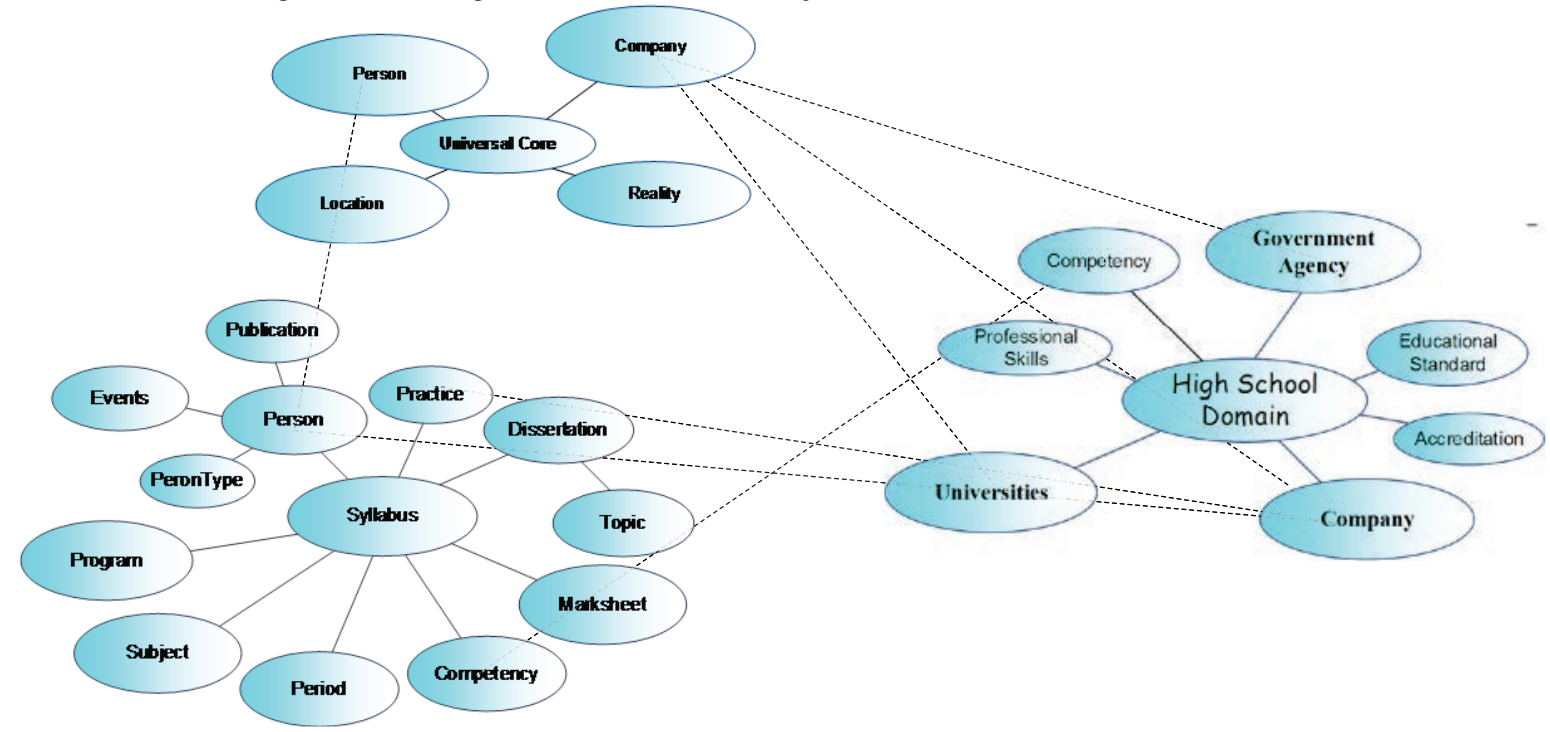

Figure 4. Bridging relationship model: Universal Core, Domain Core, Domain-Specific Core 
By using the above-described model, the opportunity arises to organize the infrastructure elements of the operation with government data, which should not only be the place where technical resources and computerization means are concentrated, but also the centers where information, knowledge, and skills are collected. The task of forming such nodes is predetermined by the need to build models for managing projects of various sorts in the field of computerization of the government sector. Skill centers should be an essential element of the infrastructure, and prospectively, factories that not only fulfill existing client queries and improve internal processes but also formulate new proposals on the information services market. Industrialization of data supply, as a tool for increasing the quality of data operations.

\subsection{CONCLUSIONS AND DIRECTIONS FOR FURTHER RESEARCH}

Based on the analysis of information models used in the world practice one may formulate principles for forming an information model of electronic government. The first principle assumes the existence of a central hub consisting of a place for collecting data from local systems. The central hub fulfills the functions of collecting and comparing data about general basic information objects and basic information objects of subject fields coming from local sources.

The second important principle consists of the fact that data for information exchange is found in the framework of managing the created information infrastructure. Data coming from agency systems should be transformed by means of components that are an integral part of the infrastructure, and after transformation should fall into the field of dissemination storage, which is also found in the area of infrastructure management.

In implementing this approach, variations are possible for distributing the responsibility for the mechanism of converting and updating data from various layers of the kernel among agencies, however organizational supervision and control at the level of data architecture, as well as quality control of the data, should be done centrally.

The fundamental advantages obtained from constructing a data model are:

the model enhances consistency and connectivity of the data;

it allows a determination of the ability to create applications of the data storage class or of supplemental nodes for operation with unified directories;

it provides improved data exchange due to semantic consistency of the data;

it provides effective data management.

There are some advantages this model under RDF: RDF technology is focused on the description of the large volume of data on a single format. Since data can be from different domains, the RFD description should be universal enough. Examples may include dictionaries FOAF, SKOS. In the proposed model suggested the division into layers: a layer of universal component layer domain and subject-specific layer, and for every layer can be applied modification procedure during usage. Also possible to add all three categories of tables: core entities, relationships between entities, as well as in the data tables. In case RDF data model to be prepared at the beginning of the project and cannot be changed during exploitation.
The RDF model is used to simulate the view of the subject area, which will be used by many participants. In the case of Data Vault domain description must meet the requirements of the problem, and is not necessarily universal.

In addition, as the advantages of this approach can be considered the fact that the majority of information technology experts work in the ideology of relational databases.

Construction of such a model will allow not only to solve tasks in the framework of a regulated data exchange but also to determine a framework for data publication on agency portals and sites, and also to create templates for data models from separate subject fields. Such approach will allow the creation of an information infrastructure that would ensure presentation of data for all possible processes in government activity.

\section{REFERENCES}

[1] RF Government Decree "On urgent measures for the creation of the state information system" Unified information environment in the sphere of systematization and coding of information " number 487 of June 1, 2016

[2] E-government System Project of the Russian Federation, minsvyaz.ru/uploaded/files/referat-ep.pdf

[3] P RF 654 r About Basic Governmental Information Resources. Order of the Government of the Russian Federation, 15 April 2011, No. 654 r, 2011.

[4] Bachilo, I. L. Methodology for systematizing functions and responsibilities in the system of federal executive authorities // Government and Law. - 2016 - No. 3. - P. 12-22.

[5] United Nations E-Government Survey http://unpan3.un.org/egovkb/Portals/egovkb/Documents/un/2 010-Survey/Chapter-6-Measuring-e-government.pdf communication

[6] Asset Description Metadata Schema (ADMS) https://joinup.ec.europa.eu/asset/adms/home

[7] National Information Exchange Model https://www.niem.gov/Pages/default.aspx

[8] Infrastructure for Multilayer Interoperability https://imi.ipa.go.jp/IMIOverview-En.html

[9] EG Infrastructure http://minsvyaz.ru/ru/activity/directions/486/

[10] Lipuntsov Y. 2015. On the Relationship Between the Information and Analytical Components in the Shared EGovernment. In Proceedings of the 2015 2nd International Conference on Electronic Governance and Open Society: Challenges in Eurasia (EGOSE '15). ACM, New York, NY, USA, 109-115.

[11] Technologies for the NIEM information model: OASIS CAM and the Open XDX Library, http://www.econ.msu.ru/science/seminars/eGoverment2.0/TecnNIEM/

[12] Inmon W.H., Linstedt D. Data Architecture: A Primer for the Data Scientist, Morgan Kaufmann , 2014.

[13] Lipuntsov Y. Principles of ontology creation for data warehouse. Example: high school // Business. Education. Law. 2016. No 3 (36). P. 72-79. 